\title{
Cell fate conversion: a chromatin remodeling checkpoint revealed
}

Cell Research (2017) 27:598-599. doi:10.1038/cr.2017.44; published online 31 March 2017

In a new paper in Cell Research, Ji et al. find that transcription factor-instigated opening of chromatin, during cell reprogramming, can be sensed by the Baf60b-containing chromatin remodeling complex, which then activates the ATM-p53 pathway, leading to cell death. These findings from reprogramming studies unveil what I term a "chromatin remodeling checkpoint" whereby extensive, inappropriate chromatin opening events lead to cell elimination, thus preventing cell fate conversion that might occur upon tissue damage; if unchecked, such conversion could lead to metaplasia and cancer.

While there is much focus today on the exciting ability to use transcription factors to change cell fates at will, it is important to understand the role and impact of cell fate changes in natural contexts. In embryonic development, newly induced transcription factors induce cell fate conversion and give rise to the diverse cell types evident in a mature organism. During adult homeostasis, transcription factors induced in stem or multipotent progenitor cells can induce new differentiated cells to replace those lost by cell turnover. However, in the face of chronic tissue injury, inappropriate transcription factor induction can induce metaplasia, where an inappropriate cell type arises in a tissue [1]. Inappropriate cell types can cause damage, as when squamous esophageal cells convert to an acid-secreting gastric cell in Barrett's esophagus, leading to chronic heartburn and a pre-cancerous condition [2]. Could there be mecha- nisms naturally resident in cells that sense when a wave of transcription factors are inappropriately expressed, thereby activating a checkpoint to impede inappropriate cell fate conversion? Such appears to be the case from a recent study from the laboratory of Lijian Hui, whereby a wave of new open chromatin sites are sensed, in response to ectopic transcription factor expression, triggering ATM-p53-based cell death [3].

In their new paper recently published by Cell Research, Ji et al. [3] ectopically expressed the liver developmental transcription factors Foxa3, HNF1 $\alpha$, and GATA4 (collectively referred to as 3TF) in mouse fibroblasts to induce hepatic conversion [4]. There is great interest in being able to generate hepatocytes in this fashion, due to the paucity of organs for liver transplantation as well as the value of generating hepatocytes in culture that accurately metabolize compounds of interest to the biomedical and pharmaceutical communities.

In the fibroblast-to-hepatic cell conversion, as in fibroblast-to-induced pluripotent stem (iPS) cell conversion, the p53 pathway gets activated, with many cells undergoing proliferation arrest and apoptosis $[3,5,6]$. Because this effect greatly reduces the efficiency of cell conversion, understanding how the p53 pathway is activated could enhance efforts to reprogram many types of cells.

To this end, Ji et al. found that the usual p53-elicited signaling pathways were not activated when fibroblasts were treated with $3 \mathrm{TF}$, but extensive ATM phosphorylation was observed
[3]. ATM is a serine/threonine kinase that can be activated by DNA doublestrand breaks and initiates a cell cycle checkpoint, leading to proliferation arrest and apoptosis. Impairment of ATM during $3 \mathrm{TF}$ treatment increases the yield of induced hepatocytes (iHeps), and impairment of p53 increases iHeps even further, while impairing either ATM or p53 alone does not induce hepatic gene expression. Interestingly, DNA doublestrand breaks and other markers of DNA damage were not activated by the 3TFs. Furthermore, knockdown of ATMIN, a protein that normally activates ATM in a DNA damage-independent fashion, impaired ATM and p53 activation by 3TF. Thus, something other than DNA damage appears to activate ATM when reprogramming factors are induced.

As expected from prior studies where reprogramming factors were shown to target silent regions of chromatin and induce locally open chromatin states $[7,8]$, Ji et al. found that closed chromatin sites targeted by $3 \mathrm{TF}$ become sensitive to a nuclease probe. Later, the regions gain histone $\mathrm{H} 3 \mathrm{~K} 9$ acetylation, associated with gene activity, and shortly afterwards, local liver genes become active. ATM activation occurs after the chromatin opening step, with phosphorylated ATM associating with newly opened chromatin sites.

With the clue about chromatin opening preceding ATM activation, Ji et al. [3] performed extensive gene knockout and knockdown studies and discovered that the SWI/SNF complex bearing the Baf60b subunit, as opposed to the complex bearing the Baf60a or Baf60c 
subunits, antagonizes iHep conversion. Yet the ATPase subunit of the Brg1 SWI/SNF complex was necessary for chromatin opening and histone H3K9 acetylation at liver target genes, whereas the Baf60b subunit was not, suggesting that Baf60a and/or Baf60c can replace $\mathrm{Baf} 60 \mathrm{~b}$ when the latter is genetically depleted. Apparently, Baf60b is necessary to recruit phosphorylated ATM to open chromatin sites via a direct interaction between ATM and Baf60b-containing Brg1 complexes.

Taken together, the findings of Ji et $a l$. suggest the presence of a "chromatin remodeling checkpoint" whereby an unusually high level of new open chromatin sites, elicited by ectopic transcription factor expression and their consequent recruitment of SWI/SNF complexes, causes the recruitment of phosphorylated ATM and activation of a p53 network for proliferation arrest and cell death. Apparently, these events occur in the absence of canonical ATMp53 pathway activation by DNA breaks.

What is the normal function of such mechanism? Why isn't it activated during natural situations where transcription factors induce new gene networks, such as in development or stem cell differentiation? Usually in the latter cases, genes are activated that relate to the parent or sister descendant cell types; thus, the total number of genes that go from a completely off to an on state (as a liver gene would during iHep conversion), may not be as frequent as genes already primed for activity. By contrast, during cell reprogramming, a much greater number of closed DNA sites are being accessed and changed [7, 8]. I suggest that cells may have evolved a "chromatin remodeling checkpoint" to respond to circumstances where an unusually high number of chromatin sites are being opened simultaneously. Such circumstance might appear during tissue damage, where key cell fate-changing factors may be activated aberrantly [2]. By sensing an unusual amount of novel chromatin opening events, the cell could be targeted for elimination and thus the tissue would avoid metaplasia. This situation may be mimicked during cell reprogramming, as unveiled by $\mathrm{Ji}$ et al. [3].

Thus, the findings of Ji et al. may have dual applications: for improving reprogramming and for a better understanding of how tissues normally maintain a cohort of cells with a common differentiation state. These views raise the question of what is the threshold of chromatin opening events that allow a cell to distinguish between inappropriate reprogramming and appropriate cell differentiation. And how does the Baf60b-containing SWI/SNF complex become engaged to activate ATM, in the absence of DNA damage? Back to the lab!

\section{Kenneth S Zaret ${ }^{1}$}

'Institute for Regenerative Medicine, Department of Cell and Developmental Biology, Perelman School of Medicine, University of Pennsylvania, 9-131 SCTR, 3400 Civic Center Blvd., Philadelphia, PA 19104-5157, USA

Correspondence: Kenneth S Zaret

Tel: 1-215-573-5813

E-mail: zaret@upenn.edu

\section{References}

1 Slack JM. Nat Rev Mol Cell Biol 2007; 8:369-378.

2 Colleypriest BJ, Burke ZD, Griffiths LP, et al. Differentiation 2017; 93:39-49.

3 Ji S, Zhu L, Gao Y, et al. Cell Res 2017; 27:642-656.

4 Huang P, He Z, Ji S, et al. Nature 2011; 475:386-389.

5 Kawamura T, Suzuki J, Wang YV, et al. $\mathrm{Na}$ ture 2009; 460:1140-1144.

6 Marión RM, Strati K, Li H, et al. Nature 2009; 460:1149-1153.

7 Soufi A, Donahue G, Zaret KS. Cell 2012; 151:994-1004.

8 Chronis C, Fiziev P, Papp B, et al. Cell 2017; 168:442-459. 